\title{
COUNTEREXAMPLE TO A CONJECTURE ON AN INFEASIBLE INTERIOR-POINT METHOD*
}

\author{
G. $\mathrm{GU}^{\dagger}$ AND C. ROOS ${ }^{\dagger}$
}

\begin{abstract}
In [SIAM J. Optim., 16 (2006), pp. 1110-1136], Roos proved that the devised fullstep infeasible algorithm has $O(n)$ worst-case iteration complexity. This complexity bound depends linearly on a parameter $\bar{\kappa}(\zeta)$, which is proved to be less than $\sqrt{2 n}$. Based on extensive computational evidence (hundreds of thousands of randomly generated problems), Roos conjectured that $\bar{\kappa}(\zeta)=1$ (Conjecture 5.1 in the above-mentioned paper), which would yield an $O(\sqrt{n})$ iteration full-Newton step infeasible interior-point algorithm. In this paper we present an example showing that $\bar{\kappa}(\zeta)$ is in the order of $\sqrt{n}$, the same order as that proved in Roos's original paper. In other words, the conjecture is false.
\end{abstract}

Key words. linear optimization, full-step infeasible interior-point algorithm, conjecture

AMS subject classifications. 90C05, 90C51

DOI. $10.1137 / 080729505$

1. Introduction. We consider the linear optimization (LO) problem in the standard form

$$
\min \left\{c^{T} x: A x=b, x \geq 0\right\}
$$

with its dual problem

$$
\max \left\{b^{T} y: A^{T} y+s=c, s \geq 0\right\}
$$

Here $A \in \mathbb{R}^{m \times n}, b \in \mathbb{R}^{m}, c \in \mathbb{R}^{n}$, and $x, s \in \mathbb{R}^{n}$ and $y \in \mathbb{R}^{m}$ are the vectors of variables. Without loss of generality we assume that $\operatorname{rank}(A)=m$.

In [1] a new infeasible interior-point method (IIPM) was proposed to solve the above LO problems. This new method can be viewed as a homotopy method. It differs from the classical IIPMs in that it uses only full steps, which has the advantage that no line searches are needed. The proven iteration bound of the algorithm is $O(n)$, and it was conjectured in [1] that this bound may be reduced to the best bound known for interior-point methods, namely, $O(\sqrt{n})$. In this paper, we construct an example showing tha, unfortunately, the conjecture is not true.

In the following, we give a brief review of the conjecture. We assume throughout that there exist an optimal solution $\left(x^{*}, y^{*}, s^{*}\right)$ and a positive number $\zeta$ such that

$$
0 \leq x^{*} \leq \zeta e, \quad 0 \leq s^{*} \leq \zeta e, \quad x^{*} s^{*}=0 .^{1}
$$

For any $\nu$ with $0<\nu \leq 1$ we consider the perturbed problem $\left(\mathrm{P}_{\nu}\right)$, defined by

$$
\min \left\{(c-\nu(c-\zeta e))^{T} x: A x=b-\nu(b-A \zeta e), x \geq 0\right\},
$$

\footnotetext{
${ }^{*}$ Received by the editors July 8, 2008; accepted for publication (in revised form) November 25, 2009; published electronically February 19, 2010.

http://www.siam.org/journals/siopt/20-4/72950.html

${ }^{\dagger}$ Faculty EEMCS, Delft University of Technology, Delft, CD 2628, The Netherlands (g.gu@ tudelft.nl, c.roos@tudelft.nl).

${ }^{1}$ Here and below, $e$ denotes the all-ones vector of length $n$, and if $x, s \in \mathbb{R}^{n}$, then $x s$ denotes the componentwise (or Hadamard) product of the vectors $x$ and $s$.
}

1862 
and its dual problem $\left(\mathrm{D}_{\nu}\right)$, which is given by

$$
\max \left\{(b-\nu(b-A \zeta e))^{T} y: A^{T} y+s=c-\nu(c-\zeta e), s \geq 0\right\} .
$$

Note that if $\nu=1$, then $x=\zeta e$ yields a strictly feasible solution of $\left(\mathrm{P}_{\nu}\right)$ and $(y, s)=$ $(0, \zeta e)$ yields a strictly feasible solution of $\left(\mathrm{D}_{\nu}\right)$. We conclude that if $\nu=1$, then $\left(\mathrm{P}_{\nu}\right)$ and $\left(\mathrm{D}_{\nu}\right)$ satisfy the IPC (interior-point condition), i.e., $\left(\mathrm{P}_{\nu}\right)$ has a feasible solution $x>0$ and $\left(\mathrm{D}_{\nu}\right)$ has a solution $(y, s)$ with $s>0$.

Let $(\mathrm{P})$ and $(\mathrm{D})$ be feasible, and let $0<\nu \leq 1$. Then problems $\left(\mathrm{P}_{\nu}\right)$ and $\left(\mathrm{D}_{\nu}\right)$ satisfy the IPC (Theorem 5.13 of [2]; see also Lemma 3.1 of [1]), and hence their central paths exist. This means that the system

$$
\begin{array}{rlrl}
b-A x & =\nu(b-A \zeta e), & x>0, \\
c-A^{T} y-s & =\nu(c-\zeta e), & & s>0, \\
x s & =\nu \zeta^{2} e & &
\end{array}
$$

has a unique solution for every $\nu>0$. In what follows this unique solution is denoted by $(x(\mu, \nu), y(\mu, \nu), s(\mu, \nu))$. These are the $\mu$-centers $\left(\mu=\nu \zeta^{2}\right)$ of the perturbed problems $\left(\mathrm{P}_{\nu}\right)$ and $\left(\mathrm{D}_{\nu}\right)$. We define

$$
\kappa(\zeta, \nu):=\frac{\sqrt{\|x(\mu, \nu)\|^{2}+\|s(\mu, \nu)\|^{2}}}{\zeta \sqrt{2 n}}, \quad 0<\nu \leq 1, \mu=\nu \zeta^{2},
$$

and

$$
\bar{\kappa}(\zeta)=\max _{0<\nu \leq 1} \kappa(\zeta, \nu)
$$

Then the total number of iterations of the full-Newton step IIPM proposed by Roos (section 4.7 of [1]) is bounded above by

$$
16 \bar{\kappa}(\zeta) \sqrt{n} \log \frac{\max \left\{n \zeta^{2},\|b-A \zeta e\|,\|c-\zeta e\|\right\}}{\epsilon} .
$$

In [1], Roos proved that

$$
\bar{\kappa}(\zeta) \leq \sqrt{2 n}
$$

which implies that the inner iteration bound is $O(n)$ (the best iteration bound for IIPMs). Based on extensive computational evidence (hundreds of thousands of randomly generated problems) Roos made the following conjecture.

Conjecture 1.1 (Conjecture 5.1 of [1]). If (P) and (D) are feasible and $\zeta \geq$ $\left\|x^{*}+s^{*}\right\|_{\infty}$ for some pair of optimal solutions $x^{*}$ and $\left(y^{*}, s^{*}\right)$, then $\bar{\kappa}(\zeta)=1$.

Clearly, the correctness of the above conjecture would reduce the iteration bound by $\sqrt{n}$.

2. Counterexample. Due to the choice of the optimal solution $\left(x^{*}, y^{*}, s^{*}\right)$, we have

$$
\begin{aligned}
& A x^{*}=b, 0 \leq x^{*} \leq \zeta e, \\
& A^{T} y^{*}+s^{*}=c, \quad 0 \leq s^{*} \leq \zeta e, \\
& x^{*} s^{*}=0
\end{aligned}
$$

Copyright $\odot$ by SIAM. Unauthorized reproduction of this article is prohibited. 
To simplify notation in the rest of this section, we denote $x:=x(\mu, \nu), y:=y(\mu, \nu)$, and $s:=s(\mu, \nu)$. Then $x, y$, and $s$ are uniquely determined by system (1.1). Using (2.1), we get the equivalent system

$$
\begin{aligned}
A x^{*}-A x & =\nu\left(A x^{*}-A \zeta e\right), & & x>0, \\
A^{T} y^{*}+s^{*}-A^{T} y-s & =\nu\left(A^{T} y^{*}+s^{*}-\zeta e\right), & & s>0, \\
x s & =\nu \zeta^{2} e . & &
\end{aligned}
$$

We rewrite this system as

$$
\begin{aligned}
A\left(x^{*}-x-\nu x^{*}+\nu \zeta e\right) & =0, & & x>0, \\
A^{T}\left(y^{*}-y-\nu y^{*}\right) & =s-s^{*}+\nu s^{*}-\nu \zeta e, & & s>0, \\
x s & =\nu \zeta^{2} e . & &
\end{aligned}
$$

Hence the maximal value that $\bar{\kappa}(\zeta)$ can attain is obtained by solving the problem

$$
\max _{0<\nu \leq 1}\left\{\frac{\sqrt{\|x\|^{2}+\|s\|^{2}}}{\zeta \sqrt{2 n}}:(2.1) \text { and }(2.2)\right\} .
$$

In this problem we maximize over all possible values of $A, b, c, \zeta, \nu, x^{*}, y^{*}, s^{*}, x, y$, and $s$ satisfying (2.1) and (2.2). Note that if (2.1) and (2.2) are satisfied, then after replacing $x^{*}, y^{*}, s^{*}, x, y, s, b$, and $c$ by $x^{*} / \zeta, y^{*} / \zeta, s^{*} / \zeta, x / \zeta, y / \zeta, s / \zeta, b / \zeta$, and $c / \zeta$, respectively, we get a solution of (2.1) and (2.2) with $\zeta=1$, and in that case the value of the objective function in (2.3) does not change. Hence, without loss of generality we may assume below that $\zeta=1$.

Our aim is to construct a feasible solution for (2.1) and (2.2) such that the objective value of (2.3) is of the same order as $\sqrt{n}$, thus showing that the order of the theoretical bound for $\bar{\kappa}(\zeta)$ in (1.2) is sharp. This will be done by first constructing suitable vectors $x^{*}, y^{*}, s^{*}, x, y, s$ such that, for some fixed value of $\nu \in(0,1)$,

$$
0 \leq x^{*} \leq e, \quad 0 \leq s^{*} \leq e, \quad x^{*} s^{*}=0, \quad x>0, \quad s>0, \quad x s=\nu e,
$$

and such that the objective value in (2.3) is of the same order as $\sqrt{n}$. After this we will construct $A, b$, and $c$ such that (2.1) and (2.2) are satisfied (for $\zeta=1$ ). It follows that the constructed $(x, y, s)$ is just the $\mu$-center of the perturbed problem pair $\left(\mathrm{P}_{\nu}\right)$ and $\left(\mathrm{D}_{\nu}\right)$ with $\mu=\nu \mu^{0}=\nu \zeta^{2}=\nu$. This will suffice to falsify Conjecture 1.1.

Using the fact that the row space of a matrix and its null space are orthogonal, we relax for the moment the first two equations in system (2.2) to

$$
\left(x^{*}-x-\nu x^{*}+\nu e\right)^{T}\left(s-s^{*}+\nu s^{*}-\nu e\right)=0, \quad x>0, \quad s>0 .
$$

Since $x^{*}$ and $s^{*}$ are orthogonal, we may rewrite the above equation as follows:

$$
x^{T}\left[\frac{1-\nu}{\nu} s^{*}+e\right]+s^{T}\left[\frac{1-\nu}{\nu} x^{*}+e\right]=(1-\nu) e^{T}\left(x^{*}+s^{*}\right)+n(1+\nu) .
$$

At this stage we choose a fixed value of $\nu \in(0,1)$ and $x^{*}$ and $s^{*}$ such that their positive entries are small enough to have

$$
\frac{1-\nu}{\nu} s^{*}+e \approx e, \quad \frac{1-\nu}{\nu} x^{*}+e \approx e, \quad(1-\nu) e^{T}\left(x^{*}+s^{*}\right)+n(1+\nu) \approx n(1+\nu) .
$$

Copyright $@$ by SIAM. Unauthorized reproduction of this article is prohibited. 
Then it follows from (2.6) that

$$
x^{T} e+s^{T} e \approx n(1+\nu) .
$$

Yet we choose

$$
x_{i}=s_{i}=\sqrt{\nu} \text { for } i>1,
$$

leaving $x_{1}$ and $s_{1}$ free for the moment. This gives

$$
x_{1}+s_{1}+2(n-1) \sqrt{\nu} \approx n(1+\nu)
$$

or, equivalently,

$$
x_{1}+s_{1} \approx(n-1)(1-\sqrt{\nu})^{2}+(1+\nu) .
$$

Our aim is to make $x$ and $s$ the $\mu$-centers of the perturbed problems corresponding to $\mu=\nu \mu^{0}=\nu \zeta^{2}$, and then to compute $\kappa(\zeta, \nu)$. This holds if $x s=\mu e$. Since $\zeta=1$, and because of (2.8), this holds if $x_{1} s_{1}=\nu$. We may easily check that there exist $x_{1}$ and $s_{1}$ which satisfy (2.9) and $x_{1} s_{1}=\nu$. Hence

$$
x_{1}^{2}+s_{1}^{2}=\left(x_{1}+s_{1}\right)^{2}-2 x_{1} s_{1} \approx\left[(n-1)(1-\sqrt{\nu})^{2}+(1+\nu)\right]^{2}-2 \nu .
$$

Thus we obtain

$$
\|x\|^{2}+\|s\|^{2} \approx\left[(n-1)(1-\sqrt{\nu})^{2}+(1+\nu)\right]^{2}-2 \nu+2(n-1) \nu .
$$

Since $\zeta=1$ this implies

$$
\kappa(1, \nu)=\frac{\sqrt{\|x\|^{2}+\|s\|^{2}}}{\sqrt{2 n}} \approx \frac{\sqrt{\left[(n-1)(1-\sqrt{\nu})^{2}+(1+\nu)\right]^{2}+2(n-2) \nu}}{\sqrt{2 n}} .
$$

Note that for fixed $\nu(0<\nu<1)$ the last expression is of the same order as $\sqrt{n}$. For example, for $\nu=1 / 4$ it equals $\sqrt{(n+16) / 32}$. Note that if $\nu$ is too small, then $(2.7)$ will not be a good approximation.

Until now the vectors $x^{*}, y^{*}, s^{*}, x, y, s$ have satisfied only (2.4) and (2.5). It remains to show that there exist $A, b$, and $c$ such that (2.1) and (2.2) are satisfied. This is easy. We take for $A$ any matrix whose row space is equal to the orthogonal complement of the linear space generated by the vector $x^{*}-x-\nu x^{*}+\nu e$. Then the vector $s^{*}-s-\nu s^{*}+\nu e$ belongs to the row space of $A$, and hence there exists a vector $y$ such that $A^{T} y=s^{*}-s-\nu s^{*}+\nu e$. Taking $y^{*}=0$, it follows that (2.2) holds. Finally, taking $b=A x^{*}$ and $c=A^{T} y^{*}+s^{*},(2.1)$ also holds. Thus we have shown the existence of a feasible solution of (2.3) for which the $\kappa(\zeta, \nu)$ has the order of $\sqrt{n}$, and hence $\bar{\kappa}(\zeta)$ will be at least of this order.

Just to add some numerical evidence to this analysis, we applied the abovedescribed construction for several values of $n$ and $\nu$. We took for $x^{*}$ and $s^{*}$ randomly generated nonnegative and orthogonal vectors, whose positive entries are uniformly distributed in $(0,1 / 1000)$. For the computation of $x_{1}$ and $s_{1}$ we used (2.6) instead of its approximation (2.9). As a consequence $x$ and $s$ are the $\mu$-centers of the perturbed problems $\left(\mathrm{P}_{\nu}\right)$ and $\left(\mathrm{D}_{\nu}\right)$ with $\mu=\nu \zeta^{2}=\nu$, and $\kappa(1, \nu)$ is well approximated by $(2.10)$. We choose $\nu=1 / 2,1 / 4,1 / 16,1 / 256$. For each value of $\nu$, we construct the example, 
and the resulting values of $\kappa(\zeta, \nu)$ and $\bar{\kappa}(\zeta)$ with $\zeta=1$ are listed in Table 2.1. ${ }^{2}$ For different values of $\nu$, we plot $\bar{\kappa}(1)$ according to $\sqrt{n}$ in Figure 2.1. From the figure, we notice that $\bar{\kappa}(1)$ increases almost linearly with respect to $\sqrt{n}$.

TABLE 2.1

Typical values of $\kappa(1, \nu)$ and $\bar{\kappa}(1)$ for some values of $n$ and $\nu$.

\begin{tabular}{|ll|cccc|}
\hline & & $\nu=1 / 2$ & $\nu=1 / 4$ & $\nu=1 / 16$ & $\nu=1 / 256$ \\
\hline$n=2^{2}$ & $\kappa(1, \nu)$ & 0.7975 & 0.7901 & 0.9860 & 1.2829 \\
& $\bar{\kappa}(1)$ & 1.0000 & 1.0000 & 1.1344 & 1.3311 \\
\hline$n=3^{2}$ & $\kappa(1, \nu)$ & 0.8089 & 0.8834 & 1.3268 & 1.8778 \\
& $\bar{\kappa}(1)$ & 1.0299 & 1.2625 & 1.6239 & 1.9334 \\
\hline$n=4^{2}$ & $\kappa(1, \nu)$ & 0.8246 & 0.9991 & 1.6927 & 2.4944 \\
& $\bar{\kappa}(1)$ & 1.1877 & 1.5560 & 2.1203 & 2.5851 \\
\hline$n=5^{2}$ & $\kappa(1, \nu)$ & 0.8444 & 1.1307 & 2.0685 & 3.1003 \\
& $\bar{\kappa}(1)$ & 1.3430 & 1.8671 & 2.6135 & 3.2163 \\
\hline$n=6^{2}$ & $\kappa(1, \nu)$ & 0.8679 & 1.2734 & 2.4515 & 3.7157 \\
& $\bar{\kappa}(1)$ & 1.5057 & 2.1871 & 3.1220 & 3.8597 \\
\hline$n=7^{2}$ & $\kappa(1, \nu)$ & 0.8950 & 1.4236 & 2.8400 & 4.3271 \\
& $\bar{\kappa}(1)$ & 1.6770 & 2.5173 & 3.6338 & 4.4846 \\
\hline$n=8^{2}$ & $\kappa(1, \nu)$ & 0.9253 & 1.5791 & 3.2273 & 4.9449 \\
& $\bar{\kappa}(1)$ & 1.8506 & 2.8389 & 4.1382 & 5.1343 \\
\hline$n=9^{2}$ & $\kappa(1, \nu)$ & 0.9584 & 1.7389 & 3.6183 & 5.5528 \\
& $\bar{\kappa}(1)$ & 2.0335 & 3.1725 & 4.6459 & 5.7512 \\
\hline
\end{tabular}

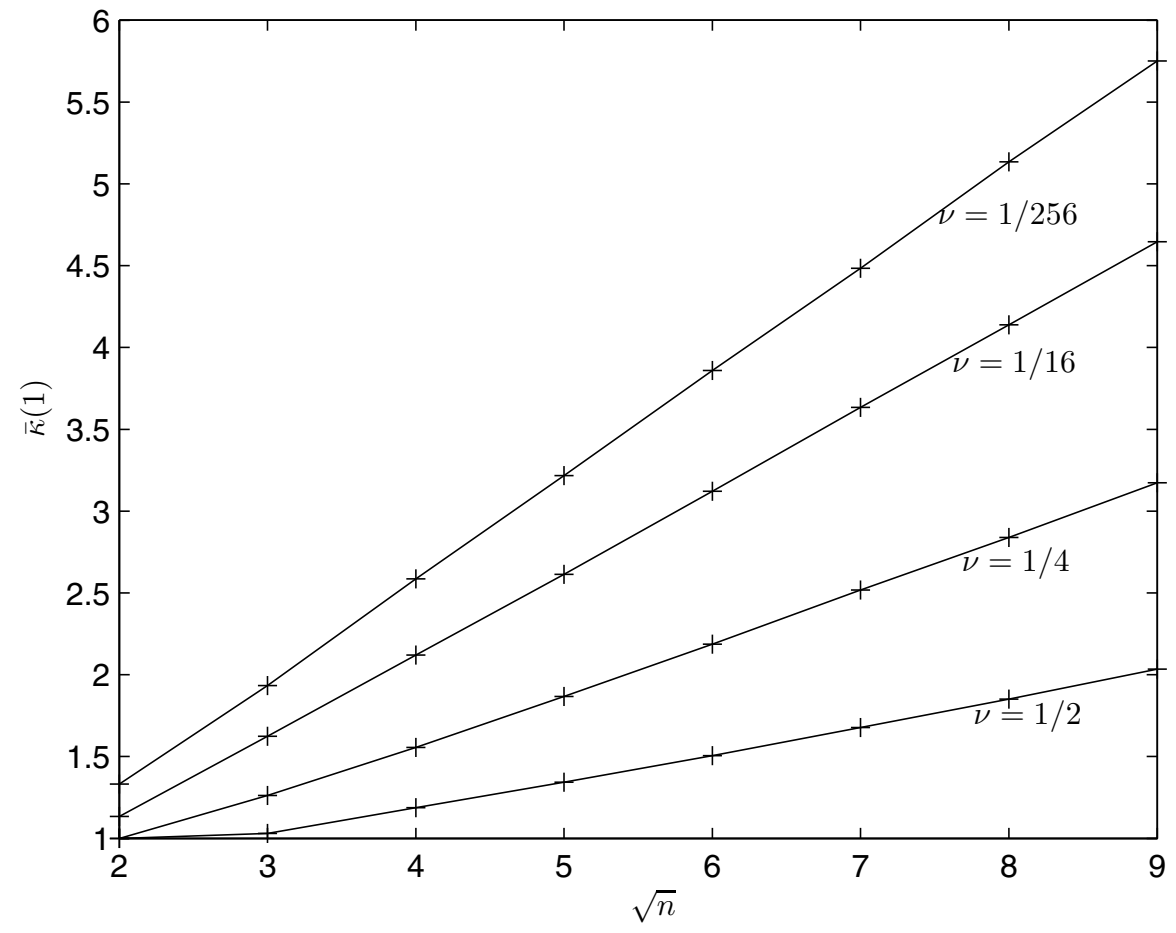

Fig. 2.1. For $\nu=1 / 2,1 / 4,1 / 16,1 / 256, \bar{\kappa}(1)$ vs. $\sqrt{n}$.

\footnotetext{
${ }^{2}$ Note that $\kappa(\zeta, \nu)$ and $\bar{\kappa}(\zeta)$ with $\zeta=1$ are denoted as $\kappa(1, \nu)$ and $\bar{\kappa}(1)$, respectively. As a consequence, we have $\bar{\kappa}(1)=\max _{0<\nu \leq 1} \kappa(1, \nu)$.
}

Copyright (C) by SIAM. Unauthorized reproduction of this article is prohibited. 
It is clear from Table 2.1 and Figure 2.1 that Conjecture 1.1 [1, Conjecture 5.1] is false.

Acknowledgments. We kindly acknowledge the useful comments of the associate editor and two anonymous referees that were very helpful in improving the exactness and readability of the paper.

\section{REFERENCES}

[1] C. Roos, A full-Newton step $O(n)$ infeasible interior-point algorithm for linear optimization, SIAM J. Optim., 16 (2006), pp. 1110-1136.

[2] Y. YE, Interior Point Algorithms. Theory and Analysis, Wiley-Intersci. Ser. Discrete Math. Optim., John Wiley \& Sons, New York, 1997. 